\title{
Nefritis intersticial granulomatosa secundario al uso del anti TNF adalimumab
}

\section{Granulomatous interstitial nephritis secondary to the use of the anti-TNF adalimumab}

\author{
César Augusto Restrepo-Valencia, Juan David Martínez-Aristizábal • Manizales (Colombia) \\ Adriana Flórez-Vargas • Bogotá, D.C. (Colombia)
}

DOI: https://doi.org/10.36104/amc.2021.2050

\begin{abstract}
Resumen
Se presenta el caso de una paciente de 56 años, quien presenta deterioro progresivo en la función renal, y en quien la biopsia renal reportó nefritis intersticial granulomatosa. Se revisó historia clínica, y se detectó que el deterioro coincidía con el inicio del medicamento adalimumab. Se suspendió, inicio terapia de esteroide más citostático con mejoría. (Acta Med Colomb 2021; 46. DOI: https:// doi.org/10.36104/amc.2021.2050).
\end{abstract}

Palabras clave: artritis reumatoidea, nefritis intersticial granulomatosa, adalimumab, anti TNF.

\begin{abstract}
We present the case of a 56-year-old patient with progressive kidney function deterioration whose kidney biopsy reported granulomatous interstitial nephritis. The medical chart was reviewed, and it was noted that the deterioration coincided with the initiation of the medication adalimumab. It was discontinued and steroid plus cytostatic treatment was begun, with improvement. (Acta Med Colomb 2021; 46. DOI: https://doi.org/10.36104/amc.2021.2050).
\end{abstract}

Key words: rheumatoid arthritis, granulomatous interstitial nephritis, adalimumab, anti-TNF.
Dr. César Augusto Restrepo-Valencia. Profesor Titular Universidad de Caldas; Juan David Martínez-Aristizábal: Residente Medicina Interna Universidad de Caldas. Manizales (Colombia); Dra. Adriana Flórez-Vargas: Patóloga Renal, Departamento de Patología y Laboratorio Clínico Hospital Universitario Fundación Santa Fe. Bogotá, D.C. (Colombia). Correspondencia: Dr. César Augusto RestrepoValencia. Manizales (Colombia) E-Mail: caugustorv@une.net.co Recibido: 19/X/2020 Aceptado: 09/II/2021

\section{Introducción}

La nefritis intersticial aguda es una patología renal en la que con poca frecuencia se piensa su existencia, a pesar de su alta incidencia. La forma granulomatosa crónica es una entidad rara y representa solo el 0.5-0.9\% de hallazgos en biopsias renales. Su etiología es amplia y ha sido asociada a diferentes tipos de medicamentos como antibióticos, AINES, enfermedades infecciosas como tuberculosis, hongos, y también a poliangeítis y sarcoidosis (1). Describimos el caso de una paciente con artritis reumatoidea, en quien se le inició terapia biológica (adalimumab), presentando deterioro progresivo y marcado de la función renal, el cual se autolimitó después de suspenderlo, descartando por hallazgos clínicos e imagenológicos otras posibilidades diagnósticas.

\section{Presentación de caso}

Paciente de 56 años, con antecedente de artritis reumatoidea seropositiva de cinco años de evolución, e hipertensión arterial crónica, quien es interconsultada a nefrología por presentar deterioro de la función renal, con un ascenso gradual y sostenido de la creatinina pasando de $1.02 \mathrm{mg} / \mathrm{dL} \mathrm{a} 2.3 \mathrm{mg} /$ dL en un lapso de nueve meses (Tabla 1), sin signos clínicos importantes, ni alteraciones macroscópicas en el examen de orina. En otros estudios se detectó anemia leve de volúmenes normales. En el uroanálisis sin proteínas, y sedimento urinario sin alteraciones en la celularidad (Tabla 2).

Se solicitó ecografía renal que reportó riñones de tamaño y aspecto normal, con adecuada diferenciación córticomedular, sin evidencia de obstrucción de las vías urinarias. En el Doppler de arterias renales no se identificó alteraciones sugestivas de estenosis de arterias renales. Se decidió solicitar biopsia renal percutánea, pero el procedimiento se autorizó y practico sólo tres meses después de la solicitud emitida por nefrología.

El resultado fue: presencia de 65 glomérulos, 29 con esclerosis global, 2 con esclerosis segmentaria, ausencia de cambios proliferativos glomerulares. En túbulointersticio inflamación $++++(100 \%)$ mononuclear con presencia de histiocitos y formación de granulomas, coloración para bacilos ácido alcohol resistentes negativa, fibrosis intersticial $+++(100 \%)$, atrofia tubular +++ (100\%), vasos sanguíneos 
Tabla 1. Niveles de creatinina en evolución clínica.

\begin{tabular}{|c|c|c|c|c|c|c|c|c|}
\hline Fecha & $4 / 23 / 2016$ & $5 / 30 / 2017$ & $8 / 25 / 2017$ & $12 / 12 / 2017$ & $2 / 12 / 2018$ & $3 / 28 / 2018$ & $\mathbf{5 / 1 9 / 2 0 1 8}$ \\
\hline Creatinina (mg/dL) & 0.5 & 1.02 & 1.25 & 1.61 & 2.3 & 2.11 & 2.03 \\
\hline \multicolumn{7}{|c|}{} \\
\hline Fecha & $\mathbf{8 / 1 7 / 2 0 1 8}$ & $\mathbf{9 / 5 / 2 0 1 8}$ & $\mathbf{1 / 2 5 / 2 0 1 9}$ & $\mathbf{4 / 1 2 / 2 0 1 9}$ & $\mathbf{2 / 1 7 / 2 0 2 0}$ & $\mathbf{7 / 1 7 / 2 0 2 0}$ & $\mathbf{9 / 2 1 / 2 0 2 0}$ \\
\hline Creatinina (mg/dL) & 3 & 2.6 & 2.19 & 2.2 & 1.97 & 1.86 & 1.9 \\
\hline
\end{tabular}

normales, inmunofluorescencia negativa para depósitos, microscopia electrónica presencia de mononucleares en intersticio, concluyéndose nefritis intersticial crónica de tipo granulomatoso (Figura 1). Se descartaron afecciones granulomatosas como tuberculosis y sarcoidosis por medio de estudios complementarios y hallazgos clínicos e imagenológicos.

Se reviso detalladamente la historia clínica, y lista de medicamentos que recibía la paciente en el momento de la evaluación: leflunomida, prednisolona, amlodipino, losartán, hidroclorotiazida, ranitidina y adalimumab $40 \mathrm{mg}$ subcutáneo cada 15 días, este último desde hacía 12 meses. Estuvo en manejo en los tres años previos con cloroquina y metotrexato. Se encontró relación entre el deterioro de la función renal y el inició de la terapia biológica (adalimumab). Con el diagnóstico histológico se optó por suspender adalimumab y leflunomida, e iniciar tratamiento con prednisona $0.5 \mathrm{mg} / \mathrm{kg} /$ día asociada a ciclofosfamida a dosis de $1 \mathrm{mg} / \mathrm{kg} /$ día por seis meses, y posteriormente continuó sólo con azatioprina, medicamento que todavía recibe a la fecha. Con la suspensión del adalimumab se detuvo el deterioro de la función renal.

\section{Discusión}

La nefritis intersticial representa un patrón de daño que se puede desarrollar por múltiples etiologías. La denominación crónica se utiliza para alguna de las diferentes entidades que en principio cursaron con una forma aguda, pero que ante la persistencia en la exposición al agente causal evolucionan a un infiltrado intersticial linfoplasmocitario, asociado a fibrosis intersticial y atrofia tubular, presentando esclerosis glomerular en estadíos más avanzados (2). La presencia de granulomas representa un patrón de daño aún más escaso, y el conocimiento sobre su etiología deriva de reportes de series de casos, donde las principales causas han sido fármacos, granulomatosis con poliangeítis, sarcoidosis y tuberculosis, variando en frecuencia dependiendo del estudio y la zona en la que se realizó $(3,4)$.

El tratamiento de enfermedades autoinflamatorias ha presentado un gran avance en los últimos años. La terapia biológica juega un papel muy importante dentro de las llamadas drogas modificadoras de la artritis reumatoide (DMARD) (5). La introducción de medicamentos como antagonistas del factor de necrosis tumoral lleva consigo el reporte posterior, luego de varios años de seguimiento, de reacciones adversas inusuales. En los últimos años se ha venido notificando la aparición de granulomas no infecciosos, no caseificantes

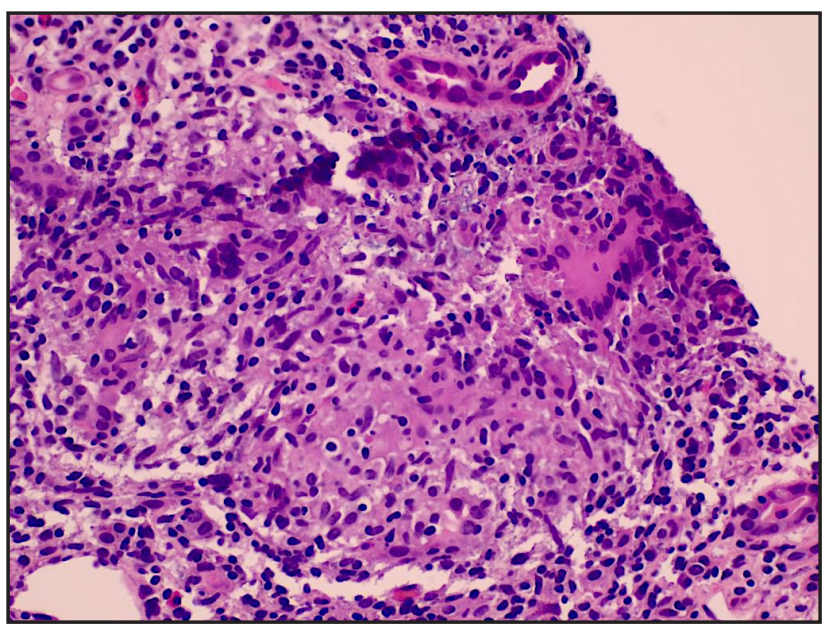

Figura 1. Hematoxilina y eosina 20x. Presencia de infiltrado inflamatorio intersticial con tendencia a formar granulomas, conformado por células histiocitoides y longitudinales.

en las biopsias renales o de ganglios linfáticos de pacientes que reciben medicamentos como adalimumab y etanercept, en los cuales se encontraba una mejoría clínica después de la suspensión del medicamento y la introducción de terapia inmunomoduladora como esteroide, situación semejante a la que ocurrió en nuestra paciente (6).

Uno de los principales diagnósticos diferenciales en el presente caso clínico, sería la sarcoidosis con compromiso renal. En esta entidad se describe que la lesión renal clásica es nefritis intersticial granulomatosa no caseificante, asociada a hipercalciuria, hipercalcemia, nefrocalcinosis y nefrolitiasis, con compromiso frecuente de otros órganos como pulmón y piel (7). En la paciente descrita se solicitó tomografía pulmonar de alta resolución, y en ella no se demostró lesiones sugestivas de sarcoidosis. El calcio sérico y en orina de 24 horas fueron reportados normales (Tabla 2).

Se descartó además la presencia de infecciones granulomatosas tipo tuberculosis, dado el patrón histológico (granulomas no caseificantes), coloración para bacilos ácidoalcohol resistentes negativo (BAAR) y prueba de tuberculina negativa, sumado a la ausencia de síntomas (Tabla 2) .

Hay pocos casos reportados en los que se documente asociación directa entre adalimumab y nefritis intersticial granulomatosa, Sandys y cols reportaron dos casos en los cuales luego de iniciar el tratamiento con dicho medicamento, se presentó un aumento gradual y sostenido en los niveles de creatinina en un periodo de aproximadamente 10 meses, la cual se estabilizó y mostró un descenso después 
Tabla 2. Otros estudios

\begin{tabular}{|c|c|}
\hline Potasio $\mathrm{mmol} / \mathrm{L}$ & 4.4 \\
\hline Ácido úrico (mg/dL) & 5.7 \\
\hline Calcio sérico total (mg/dL) & 9.8 \\
\hline Albúmina gr/dL & 4.2 \\
\hline AcVHC & Negativo \\
\hline AgsHB & 0.5 \\
\hline Elisa VIH & 0.31 (negativo) \\
\hline ANA & 1 en 80 \\
\hline $\mathbf{C 3}$ & 133 \\
\hline $\mathrm{C} 4$ & 30 \\
\hline Proteínas en orina de 24 horas (mg) & 156.3 \\
\hline Calcio en orina de 24 horas (mg) & 66 \\
\hline \multicolumn{2}{|c|}{ Examen de orina } \\
\hline Uroanálisis & $\begin{array}{l}\text { Densidad: } 1.010, \mathrm{pH}: 6.0, \\
\text { Proteínas: negativo, } \\
\text { Sangre: negativo, } \\
\text { Leucocitos: negativo }\end{array}$ \\
\hline Sedimento urinario & $\begin{array}{l}\text { Leucocitos: no se observan, } \\
\text { Eritrocitos: no se observan, } \\
\text { Células epiteliales: 1-3 CAP, } \\
\text { Bacterias: no se observan }\end{array}$ \\
\hline PPD (tuberculina) & Cero mm (negativa) \\
\hline PPD (tuberculina) & Cero mm (negativa) \\
\hline BAAR en orina & Negativo \\
\hline
\end{tabular}

de la suspensión del anti TNF (8). En los últimos años se ha venido describiendo fenómenos inflamatorios similares a la sarcoidosis (Sarcoidosis-like) secundario al uso medicamentoso anti TNF, todos los casos relacionados con la presencia de granulomas no infecciosos y no caseificantes, en biopsias de nodos linfáticos o renales, donde se sugiere que puede ser un efecto de clase, pues se ha descrito el efecto en fármacos como etanercept, infliximab y adalimumab (6). Se considera que la coexistencia de sarcoidosis y artritis reumatoide es extremadamente rara. La aparición del fenómeno de sarcoidosis-like con el uso de terapia biológica, es un efecto paradójico, dado que este tipo de tratamientos han sido utilizados con éxito en el manejo de la sarcoidosis en series de casos. Sin embargo, existe plausibilidad biológica dado la observación en cuanto a la relación temporal entre la introducción de antagonistas del TNF- $\alpha$ y la aparición de granulomatosis, con posterior mejoría del patrón inflamatorio después de que se suspenden los antagonistas del TNF- $\alpha$ y recaída clínica de la granulomatosis al redesafiar con el mismo antagonista del TNF- $\alpha$ (9).

En nuestra paciente, el retraso entre la solicitud y la práctica de la biopsia renal (tres meses) sin lugar a duda, explica que en los hallazgos histológicos se reporte fibrosis y atrofia tubular, lo que pudo repercutir en la falta de respuesta completa a la terapia inmunosupresora. A favor de ello está el reporte de Fernández-Juárez y cols, en el cual se informa que la terapia para las nefritis intersticiales agudas sólo aporta resultado satisfactorios si se inicia precozmente, antes de que se presenten cambios fibróticos (10).

Nuestra recomendación es que en pacientes con AR que presenten deterioro en la función renal y reciban terapia biológica, en especial anti TNF, se debe incluir en el diagnóstico deferencial nefritis intersticial medicamentosa, y proceder lo mas rápidamente posible a confirmarla por biopsia renal, e iniciar las terapias recomendadas, partiendo de la suspensión inmediata del anti TNF.

\section{Referencias}

1. Shah S, Carter-Monroe N, Atta MG. Granulomatous interstitial nephritis. Clin Kidney J. 2015;8(5):516-23.

2. Fogo AB, Lusco MA, Najafian B, Alpers CE. AJKD Atlas of Renal Pathology: Chronic Interstitial Nephritis. Am J Kidney Dis [Internet]. 2017;70(1):e1-2. Available from: http://dx.doi.org/10.1053/j.ajkd.2017.05.002

3. Bijol V, Mendez GP, Nosé V, Rennke HG. Granulomatous interstitial nephritis: A clinicopathologic study of 46 cases from a single institution. Int J Surg Pathol. 2006;14(1):57-63.

4. Javaud N, Belenfant X, Stirnemann J, Laederich J, Ziol M, Callard P, et al. Renal granulomatoses: A retrospective study of 40 cases and review of the literature. Medicine (Baltimore). 2007;86(3):170-80.

5. Singh JA, Saag KG, Bridges SL, Akl EA, Bannuru RR, Sullivan MC, et al. 2015 American College of Rheumatology Guideline for the Treatment of Rheumatoid Arthritis. Arthritis Care Res (Hoboken). 2016;68(1):1-25.

6. Tong D, Manolios N, Howe G, Spencer D. New onset sarcoid-like granulomatosis developing during anti-TNF therapy: An under-recognised complication. Intern Med J. 2012;42(1):89-94.

7. Correia FASC, Marchini GS, Torricelli FC, Danilovic A, Vicentini FC, Srougi M, et al. Renal manifestations of sarcoidosis: From accurate diagnosis to specific treatment. Int Braz J Urol. 2020;46(1):15-25.

8. Sandys V, Moloney B, Lane L, Qazi J, Doyle B, Barry M, et al. Granulomatous interstitial nephritis secondary to adalimumab therapy. Clin Kidney J. 2018;11(2):219-21.

9. Bhargava S, Perlman DM, Allen TL, Ritter JH, Bhargava M. Adalimumab induced pulmonary sarcoid reaction. Respir Med Case Reports [Internet] 2013;10:53-5. Available from: http://dx.doi.org/10.1016/j.rmcr.2013.07.002

10. Fernandez-Juarez G, Perez JV, Caravaca-Fontán F, Quintana L, Shabaka A, Rodriguez E, et al. Duration of treatment with corticosteroids and recovery of kidney function in acute interstitial nephritis. Clin J Am Soc Nephrol. 2018;13(12):1851-8. 